\title{
Growing InGaAs quasi-quantum wires inside semi-rhombic shaped planar InP nanowires on exact (001) silicon
}

Yu Han, Qiang Li, Shih-Pang Chang, Wen-Da Hsu, and Kei May Lau

Citation: Appl. Phys. Lett. 108, 242105 (2016); doi: 10.1063/1.4953839

View online: https://doi.org/10.1063/1.4953839

View Table of Contents: http://aip.scitation.org/toc/apl/108/24

Published by the American Institute of Physics

\section{Articles you may be interested in}

Highly ordered horizontal indium gallium arsenide/indium phosphide multi-quantum-well in wire structure on (001) silicon substrates

Journal of Applied Physics 120, 245701 (2016); 10.1063/1.4972481

Growing antiphase-domain-free GaAs thin films out of highly ordered planar nanowire arrays on exact (001) silicon

Applied Physics Letters 106, 072105 (2015); 10.1063/1.4913432

III/V nano ridge structures for optical applications on patterned $300 \mathrm{~mm}$ silicon substrate Applied Physics Letters 109, 091101 (2016); 10.1063/1.4961936

1550-nm InGaAsP multi-quantum-well structures selectively grown on v-groove-patterned SOI substrates Applied Physics Letters 111, 032105 (2017); 10.1063/1.4994318

Sub-wavelength InAs quantum dot micro-disk lasers epitaxially grown on exact $\mathrm{Si}(001)$ substrates Applied Physics Letters 108, 221101 (2016); 10.1063/1.4952600

Selective metal-organic chemical vapor deposition growth of high quality $\mathrm{GaAs}$ on $\mathrm{Si}(001)$ Applied Physics Letters 105, 062101 (2014); 10.1063/1.4892468

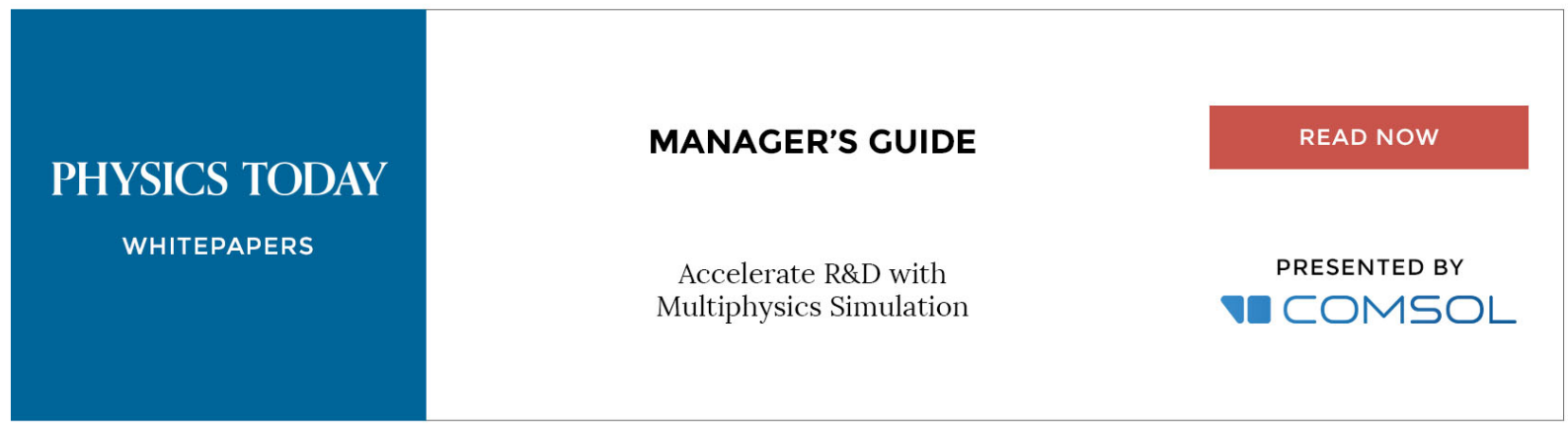




\title{
Growing InGaAs quasi-quantum wires inside semi-rhombic shaped planar InP nanowires on exact (001) silicon
}

\author{
Yu Han, ${ }^{1, a)}$ Qiang Li, ${ }^{1, a)}$ Shih-Pang Chang, ${ }^{2}$ Wen-Da Hsu, ${ }^{2}$ and Kei May Lau ${ }^{1, b)}$ \\ ${ }^{1}$ Department of Electronic and Computer Engineering, Hong Kong University of Science and Technology, \\ Clear Water Bay, Kowloon, Hong Kong \\ ${ }^{2}$ National Nano Device Laboratories, Narlabs, Hsinchu 300, Taiwan
}

(Received 22 March 2016; accepted 1 June 2016; published online 14 June 2016)

\begin{abstract}
We report InGaAs quasi-quantum wires embedded in planar InP nanowires grown on (001) silicon emitting in the $1550 \mathrm{~nm}$ communication band. An array of highly ordered InP nanowire with semirhombic cross-section was obtained in pre-defined silicon V-grooves through selective-area heteroepitaxy. The 8\% lattice mismatch between InP and Si was accommodated by an ultra-thin stacking disordered $\mathrm{InP} / \mathrm{GaAs}$ nucleation layer. X-ray diffraction and transmission electron microscope characterizations suggest excellent crystalline quality of the nanowires. By exploiting the morphological evolution of the InP and a self-limiting growth process in the V-grooves, we grew embedded InGaAs quantum-wells and quasi-quantum-wires with tunable shape and position. Room temperature analysis reveals substantially improved photoluminescence in the quasi-quantum wires as compared to the quantum-well reference, due to the reduced intrusion defects and enhanced quantum confinement. These results show great promise for integration of III-V based long wavelength nanowire lasers on the well-established (001) Si platform. Published by AIP Publishing.

[http://dx.doi.org/10.1063/1.4953839]
\end{abstract}

Monolithic integration of efficient and reliable on-chip light source on silicon remains an impediment of silicon photonics development. ${ }^{1}$ Recent advances in III-V long wavelength lasers directly grown on silicon highlight their technological importance to optical interconnects. ${ }^{2-5}$ Growing III-V planar nanostructures on patterned silicon substrates utilizing the aspect ratio trapping (ART) technique is a promising approach to develop electronic and photonic integrated circuits that incorporate optical emitters. This approach demonstrates the advantages of using large diameter industrial-standard silicon substrates, and good compatibility with the well-established silicon planar processing platform. Furthermore, ART growth could eliminate the need of thick buffers for low defect mismatched layers by employing epitaxial defect necking effect. To date, various III-V nanowires ${ }^{6,7}$ ridge quantum wells (QWs) ${ }^{8,9}$ GaAs thin films free of anti-phase domains, ${ }^{10}$ and InP distributed feedback laser array monolithically grown on (001)-silicon ${ }^{4}$ have been demonstrated.

In this letter, we report InGaAs quasi-quantum wires (quasi-QWRs) embedded in semi-rhombic InP nanowire arrays grown on exact (001) $\mathrm{Si}$ substrates using the ART method. The quantum confinement of quasi-one-dimensional electrons in quantum wires renders them attractive for applications in optical modulators, optical switches, and, particularly, semiconductor lasers. ${ }^{11,12}$ Reported research of QWRs includes selective growth on vicinal surface, ${ }^{13}$ edge QWRs by cleavage, ${ }^{14}$ and growth at the bottom of $\mathrm{V}$-grooves or at the top of ridge structure. ${ }^{15-18}$ However, these QWRs exclusively grown on III-V substrates were often accompanied by the simultaneous formation of side quantum wells (QWs).

\footnotetext{
${ }^{\text {a) }}$ Y. Han and Q. Li contributed equally to this work

b) Author to whom correspondence should be addressed. Electronic mail: eekmlau@ust.hk. Tel.: (852)23587049. Fax: (852) 23581485.
}

In this work, we demonstrate planar InP nanowires on Vgrooved (001) silicon using an ultra-thin stacking disordered nucleation layer to overcome the large lattice mismatch. InGaAs quantum structures were inserted inside the InP nanowires at varied positions. Good crystalline quality of the InGaAs quasi-QWRs near the tip of the InP nanowires and the absence of InGaAs side quantum-wells was revealed by transmission electron microscope (TEM) inspection. This allows for direct probe of the optical properties of the quasiQWRs using room temperature photoluminescence (PL) measurement. Emission from the InGaAs quasi-QWRs at the $1550 \mathrm{~nm}$ band indicates great potential of fabricating long wavelength III-V nanowire lasers on the mature Si platform.

The initial Si substrates were patterned with $150 \mathrm{~nm}$ thick $\mathrm{SiO}_{2}$ stripes along the [110] direction with a line pitch of $800 \mathrm{~nm}$ and $300 \mathrm{~nm}$ openings. After a standard RCA-1 $\left(\mathrm{NH}_{4} \mathrm{OH}: \mathrm{H}_{2} \mathrm{O}_{2}: \mathrm{H}_{2} \mathrm{O}=1: 1: 5\right)$ cleaning process and a brief $\mathrm{HF}$ dip to remove native oxide, the samples were immediately immersed in a $\mathrm{KOH}\left(45 \%\right.$ at $\left.70^{\circ} \mathrm{C}\right)$ solution to form $\mathrm{V}$-groove pockets enclosed by four $\mathrm{Si}$ (111) surfaces. Epitaxial growth was conducted in a metal-organic chemical vapor deposition (MOCVD) system with a horizontal reactor (AIXTRON 200/ 4). Triethylgallium (TEGa), tertiarybutylarsine (TBA), trimethylindium (TMIn), and tertiarybutylphosphine (TBP) were used as growth precursors and $\mathrm{H}_{2}$ as carrier gas. After an in-situ thermal cleaning process at $800{ }^{\circ} \mathrm{C}$ in $\mathrm{H}_{2}$ ambient for $15 \mathrm{~min}$, a low temperature GaAs nucleation layer was deposited at $400^{\circ} \mathrm{C}$ with a V/III ratio of 12 , followed by InP nucleation deposited at $450^{\circ} \mathrm{C}$ with a $\mathrm{V} / \mathrm{III}$ ratio of 28 . Afterwards, the reactor temperature was ramped up to $550^{\circ} \mathrm{C}$, and the bottom half of the Si pockets were filled up by growing InP at a $\mathrm{V} / \mathrm{III}$ ratio of 17 . Finally, the rest InP epilayer was grown at $600^{\circ} \mathrm{C}$ with a V/III ratio of 34 , during which $\mathrm{InGaAs}$ quantum structures were inserted. 
Fig. 1(a) shows the $70^{\circ}$ tilted-view SEM image of the planar as-grown InP nanowires separated by $\mathrm{SiO}_{2}$ stripes. Highly ordered and uniform InP nanowires with semirhombic cross-section have been obtained. The zoomed-in SEM image in Fig. 1(b) shows smooth growth facets consisting of two convex (111) B surfaces and a flat (001) surface on the top. We analyzed the epitaxial quality of the InP nanowires by high-resolution x-ray diffraction (HRXRD) measurement using an Empyrean system. The (004) plane diffraction peak of the InP nanowires, with a full-width-athalf-maximum (FWHM) of $234 \mathrm{arcsec}$, is plotted with the one from the Si substrate in the $\omega$-2theta curve (Fig. 2). To further assess the defect level, $\omega$-rocking curves across the InP peak was measured, with the line-focused $x$-ray beam in aligned orientations perpendicular or parallel to the nanowires. As shown in the inset of Fig. 2, FWHM of 659 and 731 arc sec were obtained from the parallel and perpendicular direction, respectively. These FWHM values are comparable to that measured from $1.8 \mu$ m-thick InP thin films on planar GaAs-on-Si substrates ${ }^{19}$ and slightly larger compared to $\mathrm{GaAs}^{20}$ and $\mathrm{InP}^{7}$ on $\mathrm{V}$-grooved $\mathrm{Si}$ using a higher aspect ratio nano-pattern.

High resolution TEM was performed to investigate the crystalline quality and the defect trapping mechanism in the InP nanowires. We prepared TEM specimens using conventional mechanical polishing and ion beam milling process. A JEOL2010F field-emission microscope operating at $200 \mathrm{keV}$ was used for the TEM observation. TEM images were taken along the [110] zone axis under multiple-beam conditions. We intentionally tilted the TEM specimen to achieve the best contrast to view the defects. Fig. 3(a) presents a crosssectional TEM image of a representative InP nanowire. A majority of the defects generated at the III-V/Si interface are confined within the $\mathrm{V}$-grooved pocket, with a few visible dislocations and stacking faults propagating into the bulk of the InP wire and ending at the (111) InP surface. As a result, better crystalline quality is obtained near the tip of the InP rhombic nanowire. The high resolution TEM image in Fig. 3(b) reveals a rounded growth front of the nanowire tip with a transitional (113) surface connecting the top (001) and side (111) B surfaces. The formation of these non-flat facets is due to the interfacial energy of the $\mathrm{SiO}_{2}$ sidewall and the inclination to minimize total surface energy during the selective heteroepitaxy process. Fig. 3(c) presents a zoomed-in TEM image taken at the $\mathrm{InP} / \mathrm{GaAs} / \mathrm{Si}$ interface. Stacking faults in parallel with the growth surface were formed during the low-temperature-deposited $\mathrm{InP} / \mathrm{GaAs}$ nucleation buffer to accommodate the $8 \%$ lattice mismatch. This stacking disordered layer of $\sim 15 \mathrm{~nm}$ in thickness terminates at the corner under the edge of the $\mathrm{SiO}_{2}$ stripe, as indicated by the yellow line in Fig. 3(a). A close-up of the III-V/Si interface manifests the critical role of the GaAs nucleation layer in the subsequent InP growth (Fig. 3(d)). Merckling et al. reported direct nucleation of $\mathrm{InP}$ on $\mathrm{V}$-grooved $\mathrm{Si}$ by lowering the growth temperature of the $\mathrm{InP}$ nucleation layer to $370{ }^{\circ} \mathrm{C}$ together with using a V/III ratio of larger than $2000 .^{7}$ However, it is difficult to deploy such extreme growth conditions for optimized crystalline quality. In our studies, growing $\mathrm{InP}$ in $\mathrm{Si} \mathrm{V}$-grooves without the GaAs nucleation layer resulted in large and sparsely deposited InP clusters. Another study reported by Li et al. employed thick GaAs buffer filling up the entire $\mathrm{V}$-grooved pockets. ${ }^{9} \mathrm{InP}$ was deposited on the flat (001) GaAs surface subsequently. However, such an approach undermines the unique defect trapping capability of $\mathrm{Si} \mathrm{V-grooves.} \mathrm{In} \mathrm{this} \mathrm{work,} \mathrm{we} \mathrm{initiated} \mathrm{the} \mathrm{III-} \mathrm{V} / \mathrm{Si}$ hetero-epitaxy with GaAs to promote uniform InP coverage on the Si surface. The GaAs layer was kept extremely thin so that defect trapping benefits of growing InP on (111) GaAs/ $\mathrm{Si}$ can be preserved. The lattice mismatch between InP and GaAs was accommodated by the formation of a few nanometer-thick stacking faults that ran parallel to the (111) Si surfaces instead of threading dislocations that would propagate upward.

We grew InGaAs quantum-well and quasi-quantumwire structures at various positions inside the rhombic InP nanowires by tuning the insertion time of the InGaAs layer. Three samples (QW-1, QWR-1, and QWR-2) have been prepared using the same gas flow rates. Cross-sectional TEM images in Fig. 4 show the morphological evolution from quantum-well to quasi-quantum-wire. In sample QW-1 (Fig. 4(a)), we grew an InGaAs layer flush with the V-grooved Si pocket for $60 \mathrm{~s}$. A $10 \mathrm{~nm}$ flat InGaAs quantum well with uniform thickness across the pocket opening is clearly identified in the zoomed-in TEM image in Fig. 4(b). Dislocations penetrating the quantum well and terminating at the (111) InP surface are also observed. In sample QWR-1 (Fig. 4(c)), growth of the InGaAs layer has been postponed until the formation of $\{111\}$ side facets. A crescent-shaped quasi-QWR instead of flat QW is observed. The zoomed-in TEM image in Fig. 4(d) reveals that the quasi-QWR is $20 \mathrm{~nm}$ thick and $\sim 130 \mathrm{~nm}$ wide. It is worth noting that the same growth time (60 s) with QW-1 was used for the growth of QWR-1, yet the growth rate has been doubled due to the reduced area on the $\{001\}$ plane. The TEM image in Fig. 4(d) also details that
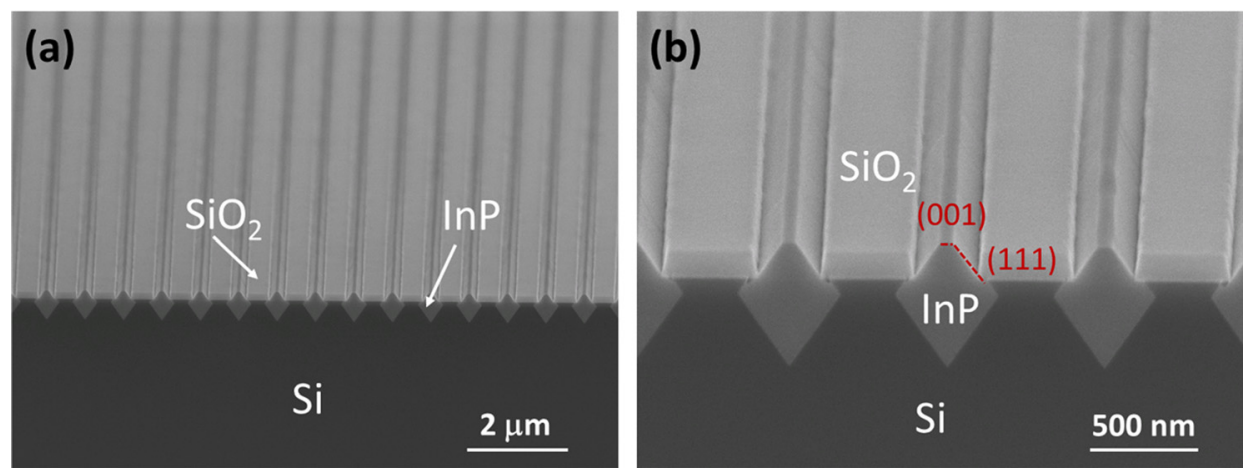

FIG. 1. (a) $70^{\circ}$ tilted SEM image of as grown highly ordered InP nanowires separated by $\mathrm{SiO}_{2}$ spacers; (b) zoomed in tilted SEM image showing the semirhombic cross-section and flat (111) and (001) InP facets. 


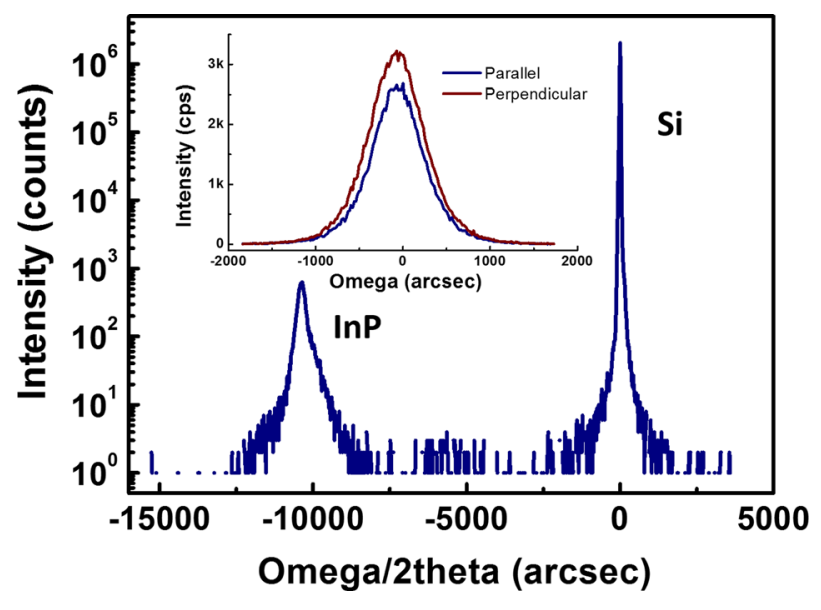

FIG. 2. Typical HRXRD $\omega$-2theta scan of the InP nanowires grown on Vgrooved Si (the Si (004) and InP (004) peaks are indicated). The inset compares $\omega$-rocking curves measured from directions perpendicular and parallel to the InP nanowires.

the quantum wire is wrapped up by thin $(\sim 10 \mathrm{~nm}) \mathrm{InP}$ on the left and the right sides and $60 \mathrm{~nm} \mathrm{InP}$ on the top. The InGaAs thickness on the $\{111\}$ facets is less than $1 \mathrm{~nm}$, indicating a large growth preference on the $\{001\}$ surface. As growth proceeds, we expect a further decrease of the $\{001\}$ surface, accompanied with an increase of nanowire tip curvature. Fig. 4(e) displays the TEM image of sample QWR-2, in which the InGaAs quantum wire was placed further up towards the tip region of the InP wires. To compensate for the enhanced growth rate, the growth time of the InGaAs layer was reduced to $9 \mathrm{~s}$. As a result, we were able to shrink the quantum-wire dimension in both vertical and lateral directions. As shown in the zoomed-in image in Fig. 4(f), a vertical thickness of $13 \mathrm{~nm}$ and a lateral width of $\sim 90 \mathrm{~nm}$ are (a)

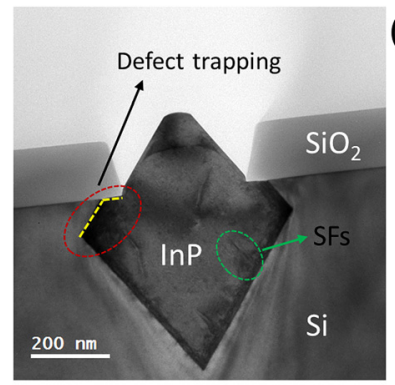

(c)

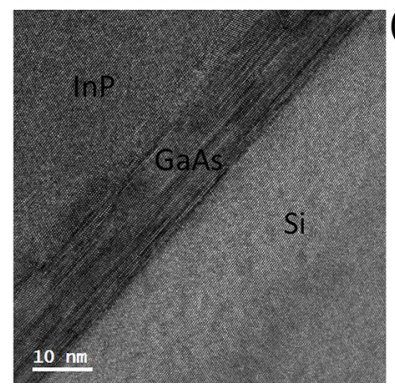

(b)

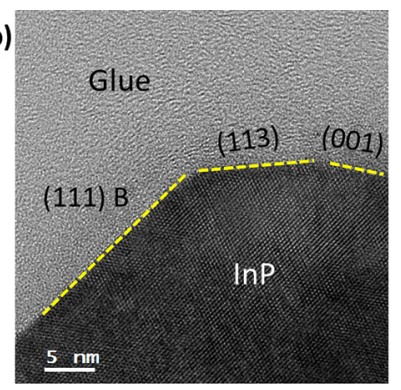

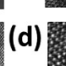

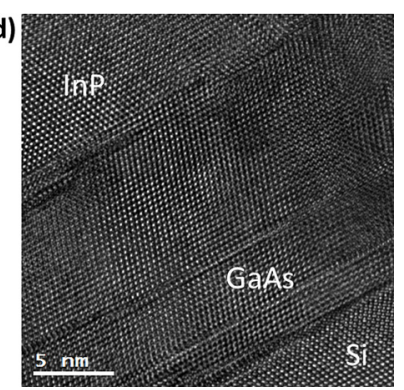

FIG. 3. (a) Cross-sectional TEM image (perpendicular to the trench direction) of a single semi-rhombic InP nanowire with labeled regions; (b) zoomed in TEM image showing the three facets at the tip of the InP nanowire: (111) B, (113), and (001); (c) cross-sectional TEM image at the InP/ $\mathrm{GaAs} / \mathrm{Si}$ interface, indicating the formation of stacking faults along the direction of the Si surface at initial nucleation stage; and (d) high resolution TEM image of the III-V/Si interface.
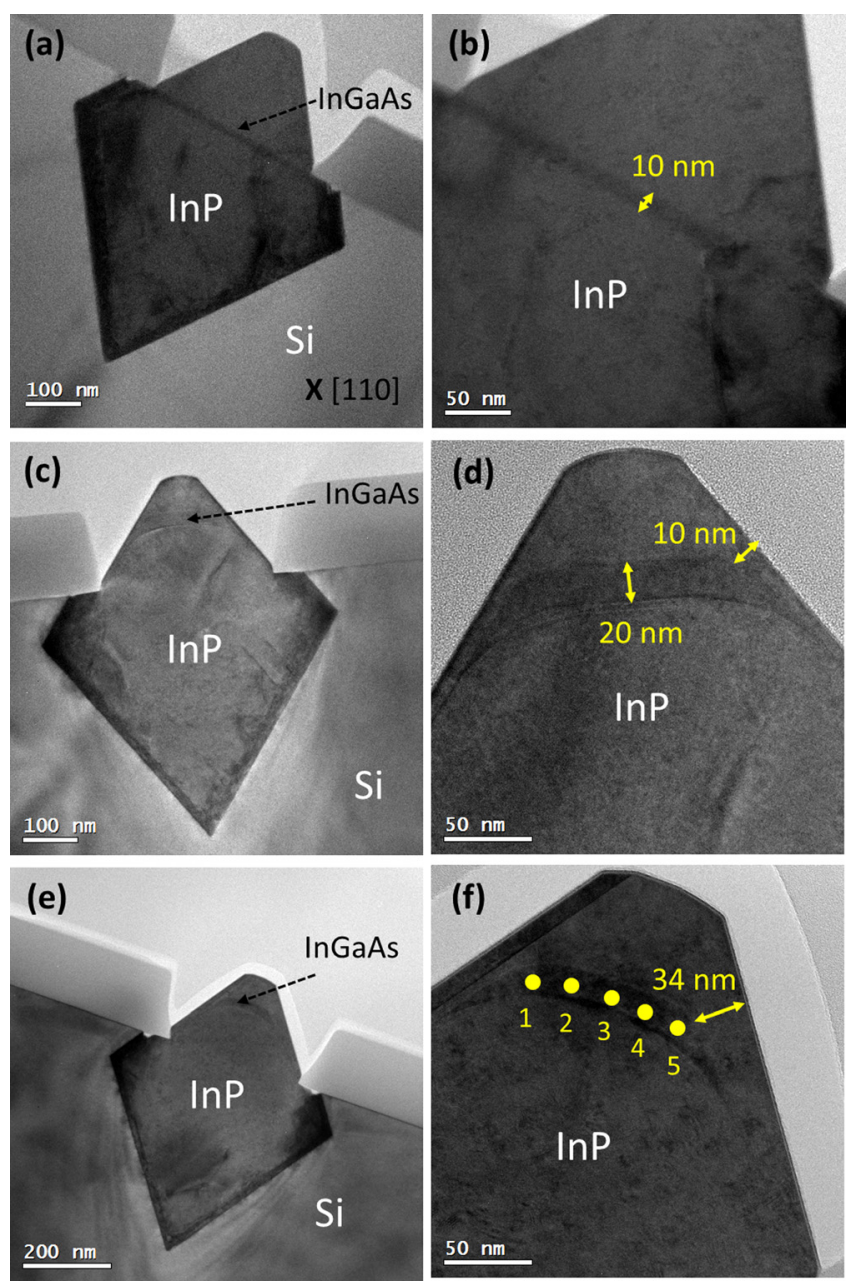

FIG. 4. (a) and (b) Cross-sectional TEM image showing the formation of the InGaAs quantum well flush with the $\mathrm{SiO}_{2}$ separating the $\mathrm{V}$-grooved pockets, and zoomed-in TEM image measuring the $10 \mathrm{~nm}$ thick InGaAs. (c) and (d) Cross-sectional TEM showing the formation of quasi-quantum wire near the top of InP nanowire and zoomed-in image indicating a $20 \mathrm{~nm}$ thick InGaAs QWR wrapped up by $10 \mathrm{~nm} \mathrm{InP}$ at lateral direction and $60 \mathrm{~nm} \mathrm{InP}$ at vertical direction. (e) and (f) High-resolution TEM image showing quasi-quantum wire with better quantum confinement at both lateral and vertical directions. The $13 \mathrm{~nm}$ thick InGaAs QWR is wrapped by $34 \mathrm{~nm}$ InP laterally and $50 \mathrm{~nm}$ InP vertically. EDX measurement points are also labeled.

obtained in QWR-2. The surrounding InP barrier has a thickness of $34 \mathrm{~nm}$ on the sides and a vertical thickness of $56 \mathrm{~nm}$ on the top.

The TEM images in Fig. 4 reveal a high degree of InGaAs thickness inhomogeneity in the quasi-QWR samples. We have further characterized the indium composition using energy-dispersive X-ray spectroscopy (EDX) from the edge to the center of the quasi-QWR in QWR-2. The measured positions are marked in Fig. 4(f). As shown in Fig. 5, indium fraction peaks at the center region of the InGaAs well and decreases as the measurement point moves to the sides. Such spatial distribution could be due to the diffusion of indium adatoms from the high curvature region (the transitional facets) to the flat region (in this case, (001) facets) due to a large diffusion coefficient. ${ }^{21}$ We therefore expect there is a lateral quantum confinement effect at the central region from the edge directions.

The optical quality of both the quantum-well and the quasi-quantum-wires was characterized by room temperature 


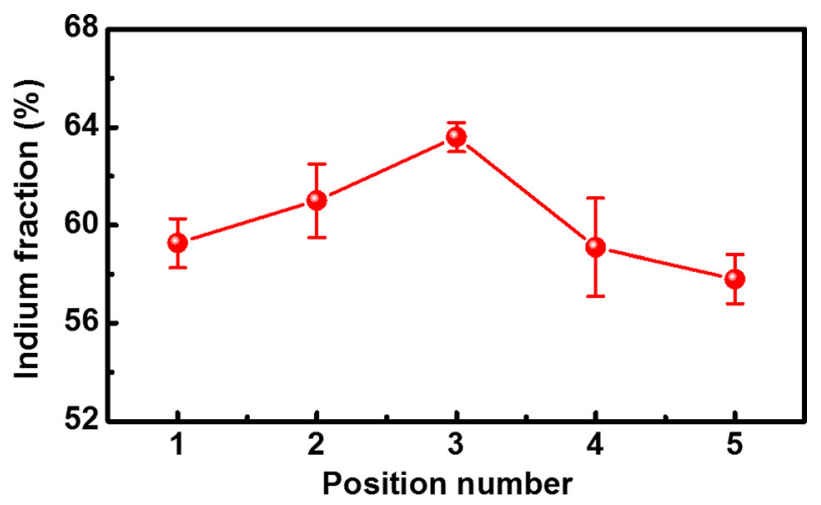

FIG. 5. Average indium fraction at different position of QWR-2 from EDX.

PL measurement using a $671 \mathrm{~nm}$ laser and a Ge detector. The excitation power was $300 \mathrm{~mW}$, and the laser spot size was around $100 \mu \mathrm{m}$ in diameter. The PL spectra of the three samples measured at full laser power are plotted in Fig. 6 for comparison. Sample QW-1 exhibits a central emitting wavelength of $1516 \mathrm{~nm}$ and a FWHM of $168 \mathrm{~nm}$. A five-fold enhancement in the peak intensity is achieved in QWR-1 as compared with QW-1, along with an emitting wavelength of $1512 \mathrm{~nm}$ and a FWHM of $170 \mathrm{~nm}$. Combined with better confinement, enhanced material quality at the upper region of the InP nanowire and reduced likelihood of defect penetration in the quantum wire led to the substantial improvement in PL emission. From QWR-1 to QWR-2, the peak PL intensity was almost doubled. The central emitting wavelength of QWR-2 was slightly shifted to a shorter wavelength of $1480 \mathrm{~nm}$, and a FWHM of $184 \mathrm{~nm}$ was obtained. To investigate the dominant mechanism behind the PL improvement, power-dependent PL measurements were performed and the results are shown in the inset of Fig. 6. Comparing the integrated PL intensity of the flat quantum well with that of the quasi-quantum wires, the difference in PL intensity decreases slightly with increasing pumping power. This reflects the influence of defect density. The lower the pump power, the more sensitive of PL intensity to defects. $^{22}$ Comparing the two QWR samples, the difference in the PL intensity increases somewhat as the pumping

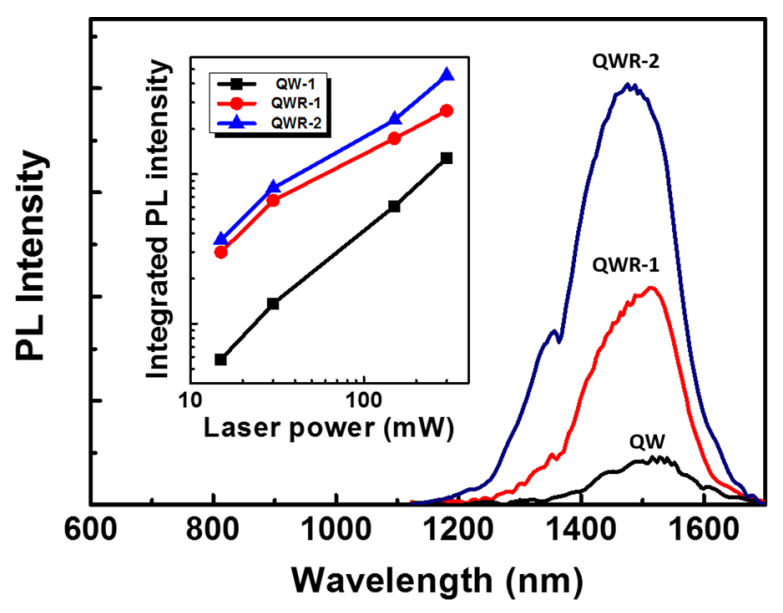

FIG. 6. Room temperature PL measurement of the three samples: QW-1, QWR-1, and QWR-2. The inset shows power dependent measurement PL of the three samples. power density increases. As the position of both QWR-1 and QWR-2 are near the top tip of the InP nanowire, we expect there is little difference in the defect densities. Enhanced quantum confinement effect in the vertical direction in QWR-2 should take most of the credit for the PL improvement (QWR-1 thickness is $20 \mathrm{~nm}$ and QWR-2 thickness is $13 \mathrm{~nm}$ ).

In conclusion, we have demonstrated the growth of InGaAs quasi-QWRs embedded in the highly ordered InP semi-rhombic nanowires on exact (001) Si substrates. Vgrooved pockets with four (111) Si interfaces were utilized to trap the defects generated at the III-V/Si interface and a mere $10 \mathrm{~nm}$ thick GaAs interlayer was used to accommodate the $8 \%$ lattice difference between InP and Si. High quality InP nanowires with rhombic cross-section and flat facets were achieved as evidenced by SEM and HRXRD measurements. Inserting InGaAs layers at different phase of the growth led to the formation of QWs and quasi-QWRs. The high crystalline quality and optical property of these InGaAs quantum structures were examined through TEM and PL measurements. The evolution of these quantum structures was accompanied by PL enhancement and a slight blue shift of the central emitting wavelength.

This work was supported in part by Grants (614813 and 16212115) from the Research Grants Council of Hong Kong. The authors would like to thank the MCPF of HKUST for technical support. Helpful discussions with C. W. Tang, C. Liu, B. Shi, and Y. Wan are also acknowledged.

${ }^{1}$ Z. P. Zhou, B. Yin, and J. Michel, Light: Sci. Appl. 4(11), e358 (2015).

${ }^{2}$ A. L. Liu, C. Zhang, J. Norman, A. Snyder, D. Lubyshev, J. M. Fastenau, A. W. K. Liu, A. C. Gossard, and J. E. Bowers, Appl. Phys. Lett. 104, 041104 (2014).

${ }^{3}$ S. M. Chen, W. Li, J. Q. Wu, M. C. Tang, S. Shutts, S. N. Elliott, A. Sobiesierski, A. J. Seeds, I. Ross, P. M. Smowton, and H. Y. Liu, Nat. Photonics 10, 307-311 (2016).

${ }^{4}$ Z. C. Wang, B. Tian, M. Pantouvaki, W. M. Guo, P. Absil, J. V. Campenhout, C. Merckling, and D. V. Thourhout, Nat. Photonics 9(12), 837-842 (2015).

${ }^{5}$ Y. Wan, Q. Li, A. Y. Liu, A. C. Gossard, J. E. Bowers, E. L. Hu, and K. M. Lau, Opt. Lett. 41(7), 1664 (2016).

${ }^{6}$ J. Z. Li, J. Bai, J.-S. Park, B. Adekore, K. Fox, M. Carroll, A. Lochtefeld, and Z. Shellenbarger, Appl. Phys. Lett. 91, 021114 (2007).

${ }^{7}$ C. Merckling, N. Waldron, S. Jiang, W. Guo, N. Collaert, M. Caymax, E. Vancoille, K. Barla, A. Thean, M. Heyns, and W. Vandervorst, J. Appl. Phys. 115, 023710 (2014).

${ }^{8}$ Q. Li, Y. Han, X. Lu, and K. M. Lau, IEEE Electron Device Lett. 37, 24-27 (2016).

${ }^{9}$ S. Li, X. Zhou, M. Li, X. Kong, J. Mi, M. Wang, W. Wang, and J. Pan, Appl. Phys. Lett. 108, 021902 (2016).

${ }^{10}$ Q. Li, K. W. Ng, and K. M. Lau, Appl. Phys. Lett. 106, 072105 (2015).

${ }^{11}$ Y. Arakawa and H. Sakaki, Appl. Phys. Lett. 40, 939 (1982).

${ }^{12}$ M. Asada, Y. Miyamoto, and Y. Suematsu, Jpn. J. Appl. Phys., Part 2 24, L95-L97 (1985).

${ }^{13}$ P. M. Petroff, A. C. Gossard, and W. Wiegmann, Appl. Phys. Lett. 45, 620 (1984).

${ }^{14}$ L. Pfeiffer, K. W. West, H. L. Stormer, J. P. Eisenstein, K. W. Baldwin, D. Gershoni, and J. Spectra, Appl. Phys. Lett. 56, 1697 (1990).

${ }^{15}$ E. Kapon, D. M. Hwang, and R. Bhat, Phys. Rev. Lett. 63, 430 (1989).

${ }^{16}$ M. Kappelt, M. Grundmann, A. Krost, V. Türck, and D. Bimberg, Appl. Phys. Lett. 68, 3596 (1996).

${ }^{17}$ C. S. Tsai, J. A. Lebens, C. C. Ahn, A. Nouhi, and K. J. Vahala, Appl. Phys. Lett. 60, 240 (1992).

${ }^{18}$ S. Koshiba, H. Noge, H. Akiyama, T. Inoshita, Y. Nakamura, A. Shimizu, Y. Nagamume, M. Tsuchiya, H. Kano, H. Sakaki, and K. Wada, Appl. Phys. Lett. 64, 363 (1994). 
${ }^{19}$ Q. Li, X. J. Zhou, C. W. Tang, and K. M. Lau, IEEE Trans. Electron Devices 60(12), 4112-4118 (2013).

${ }^{20}$ T. Orzali, A. Vert, B. O'Brien, J. L. Herman, S. Vivekanand, R. J. W. Hill, Z. Karim, and S. S. Papa Rao, J. Appl. Phys. 118, 105307 (2015).
${ }^{21}$ G. Biasiol, A. Gustafsson, K. Leifer, and E. Kapon, Phys. Rev. B 65 , 205306 (2002).

${ }^{22}$ T. H. Gfroerer, Photoluminescence in Analysis of Surfaces and Interfaces (Wiley, Chichester, 2000). 